\title{
PSFC/JA-01-29
}

\section{Impurity Transport Studies in Tokamak Edge Plasmas Using Visible Imaging}

\author{
S. Gangadhara, B. LaBombard, and the Alcator C-Mod Team
}

November 2001

\author{
Plasma Science and Fusion Center \\ Massachusetts Institute of Technology \\ Cambridge, MA 02139 USA
}

This work was supported by the U.S. Department of Energy, Cooperative Grant No. DE-FC02-99ER54512. Reproduction, translation, publication, use and disposal, in whole or in part, by or for the United States government is permitted.

Submitted for publication to IEEE Transactions on Plasma Science. 


\title{
Impurity Transport Studies in Tokamak Edge Plasmas Using Visible Imaging
}

\author{
Sanjay Gangadhara, Brian LaBombard, and the Alcator C-Mod team
}

\begin{abstract}
Understanding impurity transport in the scrape-off layer (SOL) of tokamak plasmas is a necessary piece of developing the physics basis for designing next-generation reactors. A system for inferring impurity transport parallel and perpendicular to local magnetic field lines has been developed on Alcator C-Mod using gas-injection "plumes". In this system, impurity gas is injected at a fixed position in the SOL via a reciprocating fast-scanning probe, and the resulting emission is imaged. In this paper visible light emission patterns from $\mathrm{C}^{+1}$ and $\mathrm{C}^{+2}$ ions are presented.
\end{abstract}

The transport of impurities (defined as any material which is not the primary fusion fuel) along and across magnetic field lines in the edge plasma of a tokamak reactor is of fundamental importance to reactor operation. Impurities will always be present in a reactor due to the sputtering of materials from plasma-facing components. Impurities may also be injected externally in a controlled manner for operational or diagnostic purposes. Whether those impurities remain contained near the walls or end up in the plasma core can determine both a reactor's ignition capability as well as its overall performance. In general, the prescence of impurities in the core plasma is detrimental to reactor operation, since this results in both increased radiation losses from this region (potentially leading to degradation of core energy confinement) and fuel dilution. Alternatively, the prescence of low concentrations of impurities in the edge plasma may be beneficial, since this can lead to a significant radiation of the power entering the scrape-off layer (SOL) and a spreading of this power over a large surface area. A direct means of inferring information about impurity transport in the edge plasma is to inject impurities locally into the SOL and study the resultant impurity dispersal patterns ("plumes"). In this paper, we present images of $\mathrm{C}^{+1}$ and $\mathrm{C}^{+2}$ plumes obtained from two views of the injection location.

Carbon impurities are injected, in the form of deuterated ethylene $\left(\mathrm{C}_{2} \mathrm{D}_{4}\right)$, into the edge plasma of Alcator C-Mod [1] through a reciprocating fast-scanning probe [2]. The probe is designed to inject small amounts of gas over a short time period corresponding to the time when the probe is inserted deepest in the plasma. Gas deposition is localized, allowing transport studies to be conducted as a function of position in the SOL. Typically $\sim 5 \times 10^{16} \mathrm{C}_{2} \mathrm{D}_{4}$ molecules are injected for a period of 8-10 ms over a cross-field distance of $\sim 1 \mathrm{~mm}$ [3]. The molecules then break up into their consituent atoms, which may involve the formation of molecular radicals as well as the re-deposition of material on the probe, as governed by the local plasma conditions and the surface chemistry of the probe. These atoms will then be ionized to various charge states, at rates governed by the local plasma conditions. Throughout the ionization process, the impurities will disperse in directions parallel and perpendicular to the local magnetic field, based on values of the local transport coefficients.

Coherent fiber bundles coupled to camera lenses are used to view the resulting visible light emission patterns from the two lowest charge states of carbon from two near-perpendicular directions. The bundles are optically coupled to gated, intensified CCD cameras [4] through beam-splitter systems, composed of lenses, mirrors, and bandpass interference filters. The beam-splitters are designed to allow $\mathrm{C}^{+1}$ line radiation (at $514.3 \mathrm{~nm}$ ) and $\mathrm{C}^{+2}$ line radiation (at $465.0 \mathrm{~nm}$ ) to be imaged simultaneously by each camera, with each image occupying approximately half of the CCD chip. The field-of-view for each fiber bundle is $\sim 25$ square $\mathrm{cm}$, while the overall resolution for each optical system (bundle, beamsplitter, and camera) is $\sim$ a few mm. For typical gas-injection levels, the cameras are simultaneously triggered with exposures between $\sim 0.1-1.0 \mathrm{~ms}$, depending on the camera and the specific discharge in which the plume is generated. For exposure times this short, the motion of the probe is "frozen"; nonetheless these times are still much larger than typical time scales for ionization, excitation, and 
other atomic processes. Thus the cameras are effectively taking a snap-shot of the emission pattern resulting from a steady flow of gas.

Camera images are outputted as video signals and digitized using a Matrox Corona/4 PCI video framegrabber board [5]. Signals from each camera are fed in to the different color inputs of the board ( R, G,B), allowing images from each camera to be captured synchronously. Images are stored as $640 \mathrm{x}$ 480 arrays, corresponding to the pixel dimensions of each CCD chip. The image files are transferred to a Digital Equipment Corp. (DEC) workstation for analysis, which is performed using Interactive Data Language (IDL) v5.4 [6].

An example of images collected from both cameras is shown in Figure 1. The data in these images have been smoothed over 15 pixels using a box-car smoothing algorithm located in the IDL library. For both cameras, emission from $\mathrm{C}^{+1}$ is on the bottom half of the image and emission from $\mathrm{C}^{+2}$ is on the top half. A relative shift appears between the images due to slight misalignments in the beam-splitter optics. In both images, emission from both charge states is elongated in one direction relative to the other. This direction of elongation is expected to align with local magnetic field lines since ions freely stream along the field lines. An estimate of the local magnetic field line trajectory is shown overlayed on each plume image, providing verification. $\mathrm{C}^{+2}$ images are more extended along field lines than $\mathrm{C}^{+1}$ images because of the longer ionization lifetime for the higher charge state. Asymmetries in the parallel-to-B emission are related to background parallel flows, whereas spreading and asymmetries in the cross-field emission are related to cross-field flows and diffusivity. To distinguish between the two directions, images are mapped to "field-aligned" coordinates, using a computation of the local magnetic field components from the EFIT plasma equilibrium reconstruction code [7]. A Monte Carlo impurity transport code [8] is being used to infer values of the local parallel and cross-field transport coefficients by modeling these aligned 2-D images.

In conclusion, 2-D images of emission from $\mathrm{C}^{+1}$ and $\mathrm{C}^{+2}$ ions resulting from the localized injection of deuterated ethylene into the SOL of Alcator C-Mod have been presented. Analysis in progress of the spatial structure of these images will allow information on local impurity transport coefficients to be determined.

\section{REFERENCES}

[1] I. Hutchinson, et al., "First results from Alcator C-Mod", Phys. Plasmas, 1 (1994) 1511.

[2] B. LaBombard, S. Gangadhara, et al., "A novel Tracer-Gas Injection System for Scrape-Off Layer Impurity Transport and Screening Experiments", J. Nucl. Mater., 266-269 (1999) 571.

[3] S. Gangadhara, B. LaBombard, C. MacLatchy, "Impurity Transport Experiments in the Edge Plasma of Alcator C-Mod using Gas-Injection Plumes", J. Nucl. Mater., 290-293 (2001) 598.

[4] Xybion Systems Inc., San Diego, CA. Camera models used: ISG-250 (side view), ISG-202 (top view).

[5] Matrox Electronic Systems Ltd., Quebec, Canada H9P 2T4.

[6] Research Systems Inc., Boulder, CO.

[7] L.L. Lao, et al., "Reconstruction of Current Profile Parameters and Plasma Shapes in Tokamaks", Nucl. Fus., 25 (1985) 1611.

[8] P.C. Stangeby, et al., "Monte Carlo Modelling of Impurity Ion Transport for a Limiter Source/Sink", Nucl. Fus., 28 (1988) 1945. 


\section{Side view}

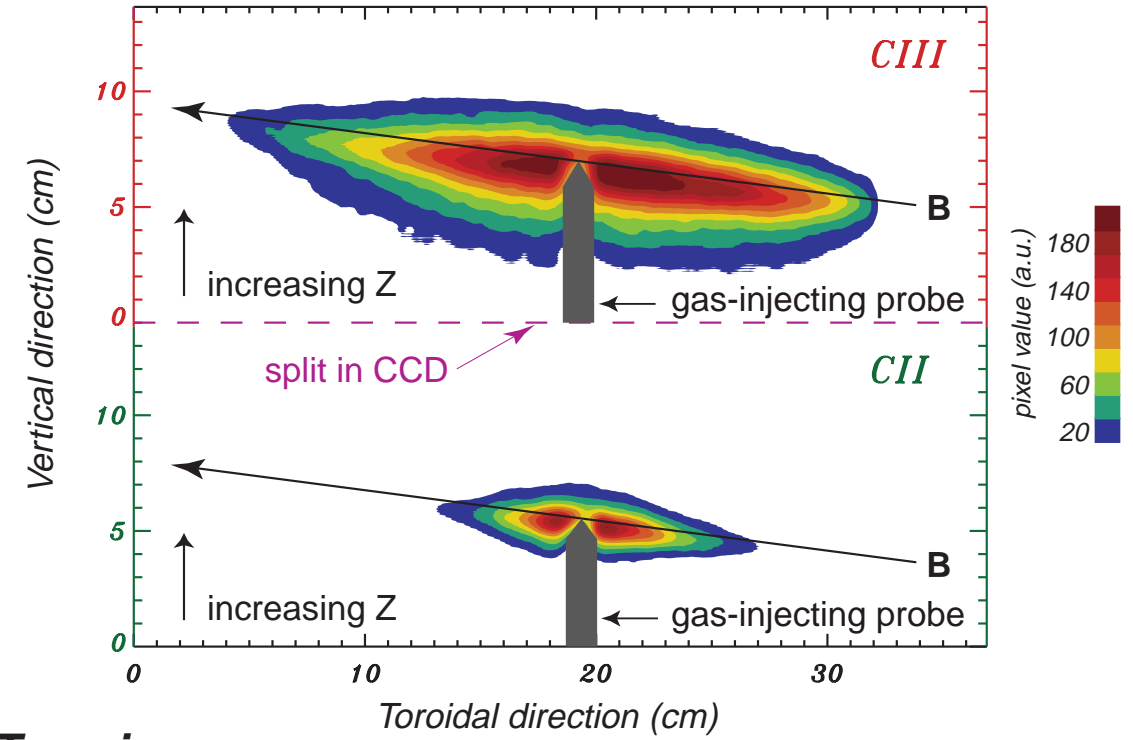

Top view

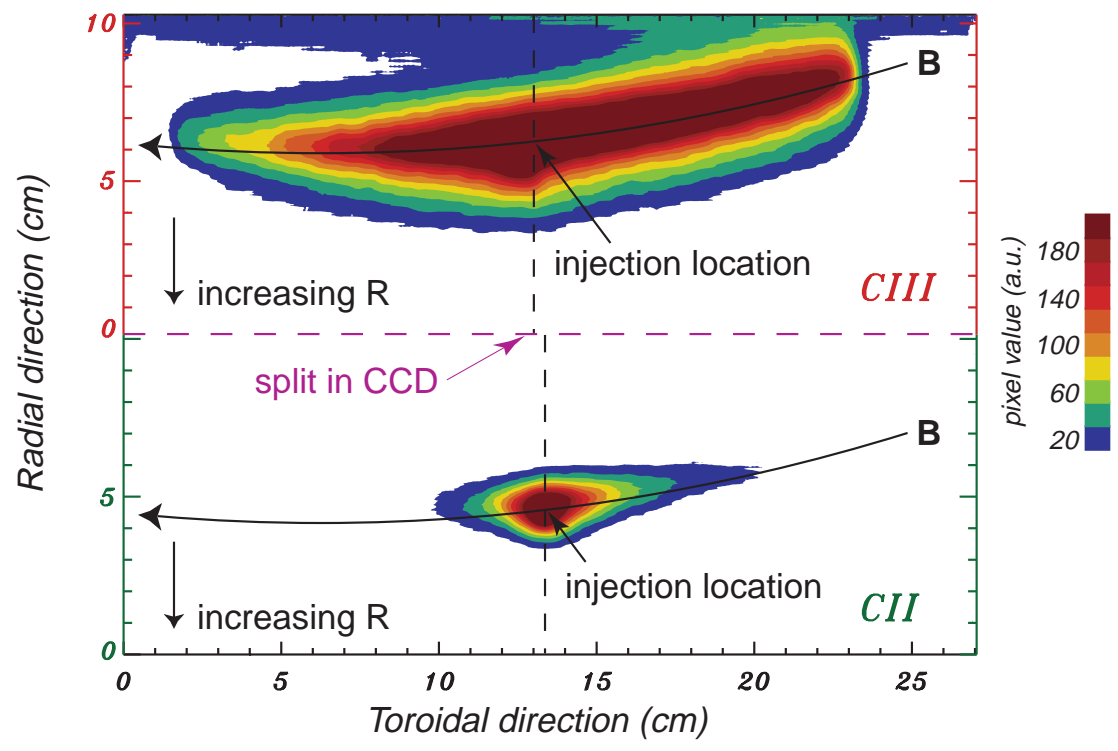

Fig. 1. $640 \times 480$ pixel CCD camera images of $\mathrm{C}^{+1}$ and $\mathrm{C}^{+2}$ emission resulting from the injection of $5 \times 10^{16}$ molecules of $\mathrm{C}_{2} \mathrm{D}_{4}$ into a background plasma with a density of $6 \times 10^{19} \mathrm{~m}^{-3}$ and a temperature of $20 \mathrm{eV}$. The side view views along the major radius of the torus, while the gas-injecting probe scans vertically. A schematic of the probe (in gray) is shown superimposed on the side view images. Emission from $\mathrm{C}^{+1}$ (green axes) and $\mathrm{C}^{+2}$ (red axes) ions is obtained simultaneously using beam-splitter optics. The horizontal axis is approximately aligned with the toroidal direction in both images, while the vertical axis approximately corresponds to the elevation (Z) for the side view and to the location in major radius $(\mathrm{R})$ for the top view. Emission is extended along field lines; an estimate of the local magnetic field line trajectory is shown overlayed on each plume image. 15 pixel smoothing has been used. 